\title{
HER2 Amplification by Next-Generation Sequencing in Lung Carcinoma: A Comparison of NGS Amplified and Non-amplified Cases by Immunohistochemistry and In Situ Hybridization
}

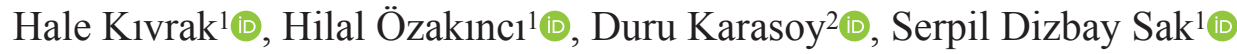

${ }^{1}$ Department of Pathology, Ankara University School of Medicine, Ankara, Turkey

${ }^{2}$ Department of Statistics, Hacettepe University, Ankara, Turkey

Background: Although the role of HER2 amplification and its evaluation methods are well known in breast carcinoma, methods for detection of HER2 amplification in non-small cell lung carcinoma are unclear. Next-generation sequencing is widely used in searching multiple therapeutic targets, and it is possible to evaluate copy number variation of genes by next-generation sequencing.

Aims: To re-evaluate the HER2 status of non-small cell lung carcinoma cases detected as HER2 amplified and non-amplified by next-generation sequencing via the most commonly used HER2 investigation methods in routine pathology practice, namely immunohistochemistry and in situ hybridization.

Study Design: Retrospective cross-sectional study.

Methods: Among the 256 patients whose mutation profiles were examined by next-generation sequencing, HER2 amplified (13 cases) and non-HER2-amplified (13 cases) were determined as study and control groups, respectively, by next-generation sequencing. HER2 next-generation sequencing amplified tumors were investigated for HER2 expression and amplification using immunohistochemistry and silver in situ hybridization.

Results: From a group of 256 non-small cell lung carcinoma, 33 tumors (12.8\%) showed HER2 amplification with next-generation sequencing. Although we observed more frequent HER2 positivity by immunohistochemistry in next-generation sequencing-amplified cases, when compared to non-amplified cases $(50 \%$ and $23 \%$ respectively), the difference was not significant $(P=.221)$. Within the HER2 amplified group, inter-method-agreement was very good between next-generation sequencing results amplification and in situ hybridization status. Next-generation sequencing results showed a strong interclass correlation coefficient with HER2/cell $(P=.009, \mathrm{r}$ $=0.777)$ and HER $2 /$ CEP17 ratio $(P=.001, \mathrm{r}=0.805)$. The median HER2/CEP17 ratio was higher in the next-generation sequencing amplified group $(P=.013)$; however, three cases were found to be amplified by silver in situ hybridization among the next-generation sequencing non-amplified cases. EGFR and FGFR1 amplification were more frequent in HER2 next-generation sequencing amplified group than next-generation sequencing non-amplified group $(P<$ $.001)$.

Conclusion: Until the effects of HER2 amplification on the HER2 protein are well understood and pulmonary carcinoma algorithms are defined, non-small cell lung carcinomas found to be amplified by nextgeneration sequencing should be verified by additional methods.

An earlier version of this study was presented orally in the 31 $1^{\text {st }}$ European Congress of Pathology (7-11 September, 2019), Nice, France

Corresponding author: Serpil Dizbay Sak, Department of Pathology, Ankara University School of Medicine, Ankara, Turkey

e-mail: Serpil.D.Sak@medicine.ankara.edu.tr

Received: February 23, 2021 Accepted: September 24, 2021 Available Online Date: January 18, 2022 • DOI: 10.5152/balkanmedj.2021.21144

Available at www.balkanmedicaljournal.org

ORCID iDs of the authors: H.K. 0000-0002-1637-7553; H.Ö. 0000-0001-7921-1000; D.K. 0000-0002-2258-4479; S.D.S. 0000-0003-3666-3095.

Cite this article as:

Kıvrak H, Özakıncı H, Karasay D, Dizbay Sak S. HER2 Amplification by Next-Generation Sequencing in Lung Carcinoma: A Comparison of NGS Amplified and Nonamplified Cases by Immunohistochemistry and In Situ Hybridization. Balkan Med J.; 2022; 39(1):21-9

Copyright@Author(s) - Available online at http://balkanmedicaljournal.org/ 


\section{INTRODUCTION}

Therapy targets in pulmonary carcinoma are extensively studied due to the high incidence and high mortality rates of this tumor. Various mutations in many genes have been identified and some of these mutations, including EGFR, ALK, ROS, and BRAF, affect the treatment protocols and prognosis of lung carcinoma patients. ${ }^{1,2}$ The necessity of both diagnosing the tumor and performing the tests for targeted therapies using a very limited amount of tissue requires careful utilization of the available material. For this reason, nextgeneration sequencing (NGS) platforms and multi-gene panels are used in many centers, to be able to detect many therapeutic targets using a single test. With the introduction of NGS platforms in many pathology laboratories, some relatively rare molecular changes with uncertain treatment consequences have started to be observed by pathologists.

Human epidermal growth receptor 2 (HER2; also known as EGFR2, ERBB2) is amplified, resulting in overexpression of the protein at the cell membrane, in $10-20 \%$ of invasive breast carcinomas. HER2 testing is required on all invasive breast carcinomas, because positive cases can be treated with HER2-targeted therapies. ${ }^{3}$ HER2 amplifications and insertions that are usually considered independent molecular events are also observed in pulmonary carcinomas and accepted as potential therapeutic targets. ${ }^{4}$ There are relatively few studies on HER2 amplification status of pulmonary carcinoma in the literature, which are performed with various methods, and the amplification/overexpression rate ranges from 0.6 to $70 \%$ (Table 1)..$^{5-9}$ There is no information about the correlation of the various methods used, and there is no gold standard concerning the test type that should be used to detect amplification in lung carcinoma. In this study, we aim to re-evaluate the HER2 status of non-small cell lung carcinoma (NSCLC) cases detected as HER2 amplified and non-amplified by NGS via the most commonly used HER2 investigation methods in routine pathology practice, namely immunohistochemistry (IHC) and in situ hybridization (ISH).

\section{MATERIAL AND METHODS}

\section{Cases}

Records and archival primary or metastatic tumor tissues from 256 NSCLC patients who consented to molecular analysis between 2018 and 2020 were used. All patients provided written informed consent. All authors ensure that the work described has been carried out in accordance with The Code of Ethics of the World Medical Association (Decleration of Helsinki). Ethics Committee of Ankara University School of Medicine awarded approval for this study (approval number: I3-160-20).

\section{Mutation Analysis}

For therapeutic decision-making, formalin-fixed paraffinembedded (FFPE) tumor tissues or fine-needle aspiration biopsy (FNAB) specimens from these patients had been previously examined for insertions/deletions, point mutations of NRAS, NTRK1, DDR2, ALK, PIK3CA, PDGFRA, KIT, RICTOR, ROS1, ESR1, EGFR, MET, BRAF, FGFR1, PTEN, KRAS, AKT1, MAP2K1, ERBB2 genes, and copy number variations (CNV) of EGFR, MET, ERBB2, FGFR1, RICTOR genes by Qiagen GeneReader NGS System (QIAGEN GeneReader Platform, Germany), using GeneRead QIAact Lung DNA UMI Panel. QCIAnalyze was used for importing the reads, trimming primers, checking quality, mapping the reads to the human reference, calling variants, and filtering on coverage. QCI-Interpretation was used for importing variants, filtering variants, and annotating with clinical consequence.

In this panel, HER2 gene region of interest for mutations was limited to 655-660 and 678-679 codons. For this reason, the most common HER2 mutation in lung cancer (codons 775-778) was not analyzed in this study.

\section{Copy Number Variation Analysis}

QCI-Interpretation program performs a coverage analysis on a number of target regions defined for the CNV target, and the observed coverage is compared to the coverage of a defined set of control samples known to not have any CNVs. There are several control steps in this method, from DNA isolation to interpretation of the results. The main quality control steps are DNA concentration, library concentration, and sequence quality control. Samples that did not provide sufficient features in these control steps were not included in this study. For each CNV target, a fold-change (FC) and a P-value are calculated. If these pass the defined thresholds, the CNV result is given as either amplification or deletion. By NGS, 33 cases were identified as HER2 amplified (NGSAmp), whereas 223 cases were non-amplified (NGSNonAmp). Residual tumor tissue in FFPE cell blocks or FFPE operation/biopsy specimens was available for further investigation, in only 13 of the 33 NGSAmp cases. These 13 cases and 13 NGSNonAmp tumors with enough tissue that were randomly selected from the 223 cases constituted our study and control groups, respectively.

\section{HER2 Immunohistochemistry}

HER2 (clone 4B5, Ventana) immunohistochemistry was performed using Ventana Bench Mark XT Autostainer (Ventana Medical Systems, Tucson, Ariz, USA) on 4- $\mu$ m-thick sections of the 13 NGSAmp and 13 NGSNonAmp tumors, along with appropriate controls, and evaluated blinded to the NGS data in accordance with the guidelines developed by American Society of Clinical Oncology/College of American Pathologists (ASCO/CAP) for breast carcinoma. ${ }^{10}$ According to this guideline, circumferential membranous staining that is complete, intense, and observed in $>10 \%$ of the tumor cells is scored as $3+$. Weak to moderate complete membrane staining observed in $>10 \%$ of the tumor cells is interpreted as $2+$. More than $10 \%$ incomplete faint membrane staining is scored as $1+$, and no staining or incomplete membrane staining that is faint/barely perceptible and observed in $\leq 10 \%$ of the tumor cells is scored as 0 .

\section{HER2 In Situ Hybridization}

In this study, $4-\mu \mathrm{m}$ thick sections of the 13 NGSAmp and 13 NGSNonAmp tumors were used for silver in situ hybridization (SISH) analysis that was performed with an automated system (INFORM, Ventana Medical Systems) using positive controls. 
Slides were stained according to the manufacturer's protocols using the INFORM HER2 dual ISH DNA cocktail (800-4422), ultraView SISH DNP Detection Kit (800-098), and ultraView Red ISH DIG Detection Kit (800-505).

Counting was performed blinded to NGS results, according to the manufacturer's interpretation guide, using an Olympus BX50 light microscope. Black signals were showing HER2 gene and red signals were showing chromosome enumeration probe 17 (CEP17). Minimum of 20 tumor cells were counted for each case. Results were categorized according to the ASCO/CAP guidelines for dual-probe HER2 ISH interpretation and HER2 was evaluated as positive if HER2/CEP17 ratio was $\geq 2.0$ and average HER2 copy number $\geq 4.0$ signals per cell. ${ }^{10}$ In situ hybridization results were accepted as 'unusual' when HER2/CEP17 $\geq 2.0$ and HER2/cell $<4.00$ or HER2/CEP17 $<2.0$ and HER2/cell was $\geq 6.00$, or HER2/ CEP17 $<2.0$ and HER2/cell was 4.0-6.00. ${ }^{11}$

\section{Statistical Analysis}

The data obtained in the study were evaluated with the SPSS (Statistical Package for Social Sciences) program version 20 for Windows (IBM SPSS Corp., Armonk, NY, USA). Continuous variables were given as median (min-max), and categorical variables were given as percentage. Mann-Whitney U-test was used to compare numerical variables of the two independent groups. Chi-square and Fisher's exact tests were used in the analysis of categorical variables. Intraclass correlation coefficient (ICC) was calculated to evaluate inter-method agreement between NGS and SISH results. Tumors that had a HER2/CEP17 ratio $>2$ were accepted as SISH positive, and the sensitivity, specificity, accuracy, positive predictive value (PPV), and negative predictive value (NPV) of NGS amplification results were calculated. Comparisons where the type 1 error level is below $5 \%(P<.05)$ were accepted as statistically significant. To evaluate the statistical power of the study, we performed post hoc power analysis, and the power rate was 0.70 when the type 1 error was taken as 0.05 .

\section{RESULTS}

\section{Patient Characteristics}

Of the 256 cases, 123 (48\%) had resections or biopsies, and 133 (52\%) had cytologic specimens (cell-blocks and/or smears). The main study group consisted of 171 adenocarcinomas (66.8\%) and

TABLE 1. Summary of studies focusing on HER2 overexpression and amplification in NSCLC in the literature

\begin{tabular}{|c|c|c|c|c|c|c|c|}
\hline & & & & $\begin{array}{c}\operatorname{HER} 2(+3) \\
\text { n }(\%)\end{array}$ & $\begin{array}{c}\operatorname{HER} 2(+2) \\
\text { n }(\%)\end{array}$ & $\begin{array}{c}\operatorname{HER} 2(+1) \\
\mathrm{n}(\%)\end{array}$ & $\begin{array}{l}\operatorname{HER} 2(0) \\
\text { n }(\%)\end{array}$ \\
\hline Study group & Number of Patients, Methods & $\begin{array}{l}\text { NGSAmp, } \\
\text { n }(\%)\end{array}$ & $\begin{array}{c}\mathrm{HER} 2 / \mathrm{Chr} 17 \geq 2 \\
\mathrm{n}(\%)\end{array}$ & \multicolumn{2}{|c|}{ HER2+ } & \multicolumn{2}{|c|}{ HER2- } \\
\hline Visscher et al..$^{9} 1997$ & $31 \mathrm{IHC}$ & & & \multicolumn{2}{|c|}{$22(70 \%)$} & \multicolumn{2}{|c|}{$9(29 \%)$} \\
\hline Hirashima et al. ${ }^{35} 2001$ & $147 \mathrm{IHC}$ & & & $1(0.6 \%)$ & $0(0 \%)$ & $20(13.6 \%)$ & $125(85 \%)$ \\
\hline Cox et al..$^{27} 2001$ & 344 IHC; 28 FISH & & $4(14.2 \%)$ & $7(2 \%)$ & $8(2.3 \%)$ & $38(11 \%)$ & $291(85 \%)$ \\
\hline Hirsch FR et al. ${ }^{36} 2002$ & $95 \mathrm{IHC}$ & & & $7(7.3 \%)$ & $26(27.3 \%)$ & $8(8.4 \%)$ & $54(56.8 \%)$ \\
\hline Tan et al. ${ }^{26} 2003$ & $131 \mathrm{IHC} ; 131 \mathrm{FISH}$ & & $7(5.3 \%)$ & \multicolumn{2}{|c|}{$25(19 \%)$} & \multicolumn{2}{|c|}{$106(81 \%)$} \\
\hline Heinmöller et al. ${ }^{5} 2003$ & $410 \mathrm{IHC} ; 378$ FISH & & $7(1.8 \%)$ & $7(1.7 \%)$ & $76(18.5 \%)$ & $86(21 \%)$ & $241(58.8 \%)$ \\
\hline Langer et al. ${ }^{16} 2004$ & $139 \mathrm{IHC}$ & & & $13(9 \%)$ & $31(22 \%)$ & $38(27 \%)$ & $50(36 \%)$ \\
\hline Clamon et al. ${ }^{17} 2005$ & $209 \mathrm{IHC}$ & & & \multicolumn{2}{|c|}{$24(11 \%)$} & \multicolumn{2}{|c|}{$185(89 \%)$} \\
\hline Cappuzzo et al. ${ }^{34} 2005$ & 72 IHC; 110 FISH & & $23(22.8 \%)$ & \multicolumn{2}{|c|}{$5(7 \%)(\mathrm{H}$ score $\geq 200)$} & \multicolumn{2}{|c|}{-} \\
\hline Kuyama et al. ${ }^{28} 2008$ & $68 \mathrm{IHC}$ & & $7(10 \%)$ & \multicolumn{2}{|c|}{$16(24 \%)(1+, 2+)$} & \multicolumn{2}{|l|}{$45(66 \%)$} \\
\hline Varella-Garcia et al. ${ }^{33} 2009$ & $44 \mathrm{FISH}$ & & $23(53 \%)$ & & & & \\
\hline Yu et al. ${ }^{14} 2013^{*}$ & 24 FISH & & $2(8.3 \%)$ & & & & \\
\hline Yoshizawa et al. ${ }^{24} 2014$ & 243 IHC; 243 FISH; 243 DISH & & $5(2.1 \%) ; 9(3.7 \%)$ & $6(2.5 \%)$ & $31(12.7 \%)$ & $103(42.3 \%)$ & $103(42.3 \%)$ \\
\hline Suzuki et al. ${ }^{7} 2015$ & 1266 IHC; 1170 BF-ISH & & $222(19 \%)$ & \multicolumn{2}{|c|}{$31(2.4 \%)$} & \multicolumn{2}{|c|}{-} \\
\hline Li et al. ${ }^{4} 2016$ & $25 \mathrm{IHC} ; 175$ FISH & & $5(3 \%)$ & $0(0 \%)$ & $0(0 \%)$ & $0(0 \%)$ & $25(100 \%)$ \\
\hline Kim et al..$^{25} 2017$ & $321 \mathrm{IHC} ; 321 \mathrm{SISH}$ & & $46(14.3 \%)$ & $4(1.2 \%)$ & $21(6.5 \%)$ & $48(15 \%)$ & $248(77.3 \%)$ \\
\hline Hotta et al. ${ }^{6} 2017$ & $15 \mathrm{IHC}$ & & & $5(33 \%)$ & $3(20 \%)$ & \multicolumn{2}{|c|}{$7(47 \%)$} \\
\hline TCGA $^{2} 2014$ & 230 NGS & $2(0.9 \%)$ & & & & & \\
\hline Lee et al. ${ }^{20} 2020$ & 1108 NGS & $15(1.3 \%)$ & & & & & \\
\hline
\end{tabular}


$85(33.2 \%)$ cases diagnosed as NSCLC-Not otherwise specified (NOS). HER2 amplification was detected in $12.8 \%$ of the 256 NSCLCs by NGS. There was no statistically significant difference between the NGSAmp and NGSNonAmp groups regarding the diagnosis $(P=.418)$, age $(P=.944)$, or sex $(P=.413)$ (Table 2$)$.

\section{Accompanying Molecular Changes}

HER2 amplifications were accompanying other pathological molecular changes in 28 of the 33 cases (85\%), EGFR amplifications being the most frequent (25 cases), followed by FGFR1 amplifications (16 cases), MET amplifications (14 cases), KRAS point mutations ( 5 cases), ALK rearrangements (3 cases), EGFR deletions/point mutations (3 cases), and a single KIT mutation. We did not observe a statistically significant difference between the NGSAmp and NGSNonAmp groups with regard to accompanying molecular abnormalities, except for the EGFR and
FGFR 1 amplifications that were present in $76 \%$ and $50 \%$ of HER2 NGSAmp cases, respectively $(P<.001$ and $P<.001)$. HER2 amplification was the single molecular change in only 5 patients, constituting $15 \%$ of the NGSAmp group and only $2 \%$ of the whole group (Table 2).

\section{Immunohistochemical HER2 Expression Versus HER2 Amplification by NGS}

In 3 of the 13 NGSAmp cases, immunohistochemical analysis was not successful due to inadequate tumor tissue; hence, only 10 NGSAmp and 13 NGSNonAmp cases could be evaluated by IHC (Table 3$)$. Five out of 10 cases from the NGSAmp group (50\%) and 10 cases out of $13(76 \%)$ from the NGSNonAmp group were immunohistochemically negative $(0,1+)$, whereas 5 and 3 cases were positive $(2+, 3+)$ in NGSAmp and NGSNonAmp groups, respectively (Figure 1a-c). Interestingly, 5 cases $(21.7 \%)$ showed

TABLE 2. Clinical and molecular characteristics of NGS-amplified and non-amplified cases

\begin{tabular}{|c|c|c|c|c|}
\hline & & $\begin{array}{c}\text { NGSAmp, } \\
\mathrm{n}=33,12.8 \%\end{array}$ & $\begin{array}{l}\text { NGSNonAmp, } \\
\mathrm{n}=223,87.1 \%\end{array}$ & $P$ \\
\hline \multicolumn{5}{|l|}{ Patients } \\
\hline \multicolumn{2}{|l|}{ Age years, mean (range) } & $63.3(37-87)$ & $63.4(37-89)$ & .944 \\
\hline \multicolumn{2}{|l|}{ Sex $M / F$} & $26(79 \%) / 7(21 \%)$ & $159(71 \%) / 64(29 \%)$ & .413 \\
\hline \multicolumn{5}{|l|}{ Tumor type } \\
\hline \multicolumn{2}{|l|}{ Adenocarcinoma } & $20(61 \%)$ & $151(68 \%)$ & .418 \\
\hline \multicolumn{2}{|l|}{ NSCLC, NOS } & $13(39 \%)$ & $72(32 \%)$ & \\
\hline \multicolumn{5}{|c|}{ Accompanying copy number variations by NGS } \\
\hline \multirow[t]{2}{*}{ EGFR } & $\mathrm{n}, \%$ & $25(76 \%)$ & $91(41 \%)$ & $<.001$ \\
\hline & FC median & $2.12(1.53-3.82)$ & $2.04(1.42-24.42)$ & .304 \\
\hline \multirow[t]{2}{*}{ FGFR1 } & $\mathrm{n}, \%$ & $16(50 \%)$ & $19(8.5 \%)$ & $<.001$ \\
\hline & FC median & $2.45(1.52-11.53)$ & $3.28(1.47-21.85)$ & .610 \\
\hline \multirow[t]{2}{*}{ MET } & $\mathrm{n}, \%$ & $14(42.4 \%)$ & $55(25 \%)$ & .105 \\
\hline & FC median & $2.69(1.76-15.60)$ & $2.26(1.43-23.97)$ & .041 \\
\hline \multicolumn{5}{|c|}{ Accompanying point mutations and small insertions/deletions by NGS } \\
\hline \multicolumn{2}{|l|}{$\operatorname{EGFR}(\mathrm{n}, \% \mathrm{~m} / \mathrm{w})$} & $3(9 \%) / 30(91 \%)$ & $34(15 \%) / 189(85 \%)$ & .436 \\
\hline \multicolumn{2}{|l|}{ KRAS (n, \% m/w) } & $5(15 \%) / 28(85 \%)$ & $54(23 \%) / 169(76 \%)$ & .375 \\
\hline \multicolumn{2}{|l|}{ NRAS (n, \% m/w) } & $0(0 \%) / 33(100 \%)$ & $1(0.3 \%) / 222(98.7 \%)$ & 1.00 \\
\hline \multicolumn{2}{|l|}{$\operatorname{PIK} 3 C A(n, \%$ m/w) } & $1(3 \%) / 32(97 \%)$ & $14(6 \%) / 209(94 \%)$ & .701 \\
\hline \multicolumn{2}{|l|}{ BRAF (n, \% m/w) } & $0(0 \%) / 33(100 \%)$ & $9(4 \%) / 214(96 \%)$ & .609 \\
\hline \multicolumn{2}{|l|}{$\operatorname{KIT}(\mathrm{n}, \% \mathrm{~m} / \mathrm{w})$} & $1(3 \%) / 32(97 \%)$ & $3(1 \%) / 220(99 \%)$ & .426 \\
\hline \multicolumn{2}{|l|}{$\operatorname{ALK}(\mathrm{n}, \% \mathrm{~m} / \mathrm{w})$} & $0(0 \%) / 33(100 \%)$ & $1(0.5 \%) / 222(99.5 \%)$ & .477 \\
\hline \multicolumn{5}{|c|}{ Gene rearrangements by immunohistochemistry and FISH } \\
\hline \multicolumn{2}{|l|}{$\mathrm{ALK}^{*} \mathrm{n}+/-$} & $3(10 \%) / 27(90 \%)$ & $10(5 \%) / 197(94.5 \%)$ & .3 \\
\hline \multicolumn{2}{|l|}{$\mathrm{ROS} 1 * \mathrm{n}+/-$} & $0(0 / \%) / 27(100 \%)$ & $3(1.5 \%) / 198(98.5 \%)$ & 1.00 \\
\hline
\end{tabular}

*ALK and ROS1 rearrangements were evaluated in 237 and 228 cases, respectively.

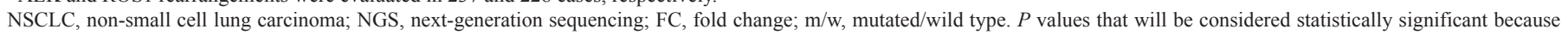
they are below 0.05 are written bold. 
a prominent cytoplasmic and/or nuclear staining that is quite rare in breast carcinomas (Figure 1d). Although we observed more frequent $2+$ or $3+$ positivity in NGSAmp cases $(50 \%)$, when compared to the NGSNonAmp group (23\%), the difference was not statistically significant $(P=.221)$.

\section{ISH Versus HER2 Amplification by NGS}

The minimum tumor percentage rate was $10 \%$ for NGSAmp and $20 \%$ for NGSNonAmp cases, respectively. Maximum tumor necrosis rate was $30 \%$ for NGSAmp and $40 \%$ for NGSNonAmp cases, respectively (Supplementary Table S1).

In 3 of the 13 NGSAmp cases, SISH analysis was not successful due to the inadequacy of tumor tissue; hence, 10 NGSAmp and 13 NGSNonAmp cases could be evaluated by SISH (Table 3). In a total of 7 cases, 3 of them being NGSNonAmp, HER2/CEP17 ratio was $\geq 2.0$ and HER2/cell ratio was $\geq 4$.0, which is the HER2positive ISH category (ISHAmp) defined for breast carcinomas (Figure 1c). Two NGSAmp and 7 NGSNonAmp cases were HER2 negative (ISHNonAmp) by breast carcinoma standards (HER2/ CEP17 <2.0, HER2/cell <4.0). Results of 4 NGSAmp and 3 NGSNonAmp cases corresponded to one of the unusual HER2 ISH result categories defined for breast carcinomas. Within the NGSAmp group, inter-method agreement was very good between NGS amplification status and ISH status. When HER2/cell ratio $>4$ was excepted as ISH positive, NGS and ISH showed a strong $\operatorname{ICC}(P=.009, \mathrm{r}=0.777)$. Similarly, when HER2/CEP17 ratio $>2$ was excepted as ISH positive, NGS and ISH showed a strong ICC $(P=.001, \mathrm{r}=0.805)$.

The median HER2/CEP17 ratio was found to be higher in NGSAmp cases when compared to NGSNonAmp cases $(P=.013$, median 2.33 vs 1.34 ).

Within the NGSAmp group, inter-method agreement was good between HER2 FC and HER2/cell ratio, and the ICC was 0.777 (95\% CI: 0.051-0.949, $P=.009$ ). HER2/cell ratio was higher in the NGSAmp group, though statistically not significant $(P$ $=.066$, median 4.95 vs 2.80 ). When NGS and ISH results were compared, NGS had a sensitivity and specificity of $66 \%$ and $76 \%$, respectively; accuracy was $72.7 \%$, positive predictive value (PPV) was $66.7 \%$, and negative predictive value (NPV) was $76.9 \%$.

\section{ISH Versus Immunohistochemical HER2 Expression}

Twenty-one patients were analyzed with both SISH and IHC. Fifty percent of SISH positive (HER2/CEP17 ratio $>2$ ) cases were positive with IHC $(2+, 3+)$. In the HER2-SISH negative group (HER2/CEP17 ratio <2), HER2-IHC positivity was determined in $23 \%$ of patients. Immunohistochemical HER2 positivity frequency was not significant in HER2-SISH positive and HER2-SISH negative groups $(P=.346)$.

One case in the NGSAmp group and 6 cases in the NGSNonAmp group were negative with both methods (IHC: 0/1+ and HER2/ CEP17 $<2.0$, HER2/cell $<4.0$ by SISH). Three cases in the

TABLE 3. Immunohistochemistry and in-situ hybridization results of NGS-amplified and non-amplified cases

\begin{tabular}{|c|c|c|c|c|c|c|c|c|}
\hline NGSAmp & Fold change & $\mathrm{IHC}$ & HER2/CEP17, HER2/cell & ISH Result & NGS NonAmp & $\mathrm{IHC}$ & HER2/CEP17, HER2/cell & ISH Result \\
\hline 1 & Mar.43 & $1+$ & $2.33,3.5$ & $\mathrm{U}$ & 1 & $1+$ & $1.91,4.4$ & $\mathrm{U}$ \\
\hline 2 & Aug. 81 & $3+$ & $7,16.15$ & + & 2.Jan & $2+$ & $1.09,2.4$ & - \\
\hline 3 & 5.Feb & $2+$ & $2.34,5.52$ & + & 3.Jan & $0^{*}$ & 1,2 & - \\
\hline 4 & 2 & 0 & $1.46,4.95$ & $\mathrm{U}$ & 4 & 0 & 1,2 & - \\
\hline 5 & May.78 & $1+*$ & $2.37,6.65$ & + & 5.Jan & $3+$ & $1.62,6.9$ & $\mathrm{U}$ \\
\hline 6 & Feb.73 & $\mathrm{TE}$ & $2.17,2.5$ & $\mathrm{U}$ & 6.Jan & 0 & $1.09,2.35$ & - \\
\hline $7 * *$ & Nov.72 & 0 & $1.25,3.65$ & - & 7.Jan & $0^{*}$ & $2.17,5.65$ & + \\
\hline 8 & Jan.63 & $2+$ & $1.81,4.9$ & $\mathrm{U}$ & 8.Jan & 0 & $1.34,2.75$ & - \\
\hline 9 & Feb.37 & $1+*$ & $\mathrm{TE}$ & $\mathrm{TE}$ & 9.Jan & 0 & $2.05,4.1$ & + \\
\hline 10 & 6.Nov & $3+$ & $4.82,9.65$ & + & 10 & $0^{*}$ & $0.88,2.7$ & - \\
\hline 11 & Jun.97 & $2+$ & $\mathrm{TE}$ & $\mathrm{TE}$ & Jan.00 & $2+$ & $2.71,5.7$ & + \\
\hline 12 & Mar.67 & $\mathrm{TE}$ & $1.27,3.45$ & - & 12.Jan & 0 & $1.08,2.8$ & - \\
\hline 13 & Mar.75 & $\mathrm{TE}$ & $\mathrm{TE}$ & $\mathrm{TE}$ & 13.Jan & 0 & $1.6,4.8$ & $\mathrm{U}$ \\
\hline Results & \multicolumn{4}{|c|}{ IHC: 5 cases $0 / 1+, 3$ cases $2+, 2$ cases $3+, 3$ cases TE } & Results & \multicolumn{3}{|c|}{ IHC: 10 cases $0 / 1+, 2$ cases $2+, 1$ cases $3+$} \\
\hline & \multicolumn{4}{|c|}{ SISH: 4 cases,+ 2 cases,- 4 cases $U, 3$ cases TE } & & \multicolumn{3}{|c|}{ SISH: 3 cases,+ 7 cases,- 3 cases $U$} \\
\hline
\end{tabular}

*Nuclear and cytoplasmic staining.

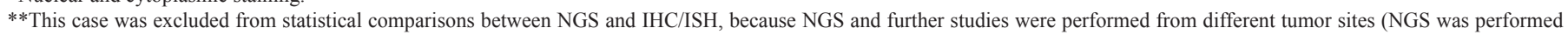
from tumor aspiration FNAB smear and IHC/SISH was performed pleural effusion cell block) that may represent different tumor sub-clones.

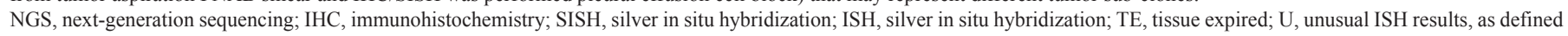
for breast carcinoma. 
NGSAmp group and 1 case in the NGSNonAmp group were in accordance with both methods and evaluated as positive (IHC: $2+/ 3+$ and HER $2 /$ CEP $17 \geq 2.0$ and HER $2 /$ cell $\geq 4.0$ by SISH). One additional NGSNonAmp case was $2+$ with IHC and ISHNonAmp.

\section{DISCUSSION}

HER2 amplification is an important therapy target in breast carcinoma, and many laboratories use IHC as the initial test. If there is an equivocal result ( $2+$ staining), the case is referred to ISH.10 Anti-HER2 treatment has been demonstrated to increase survival in HER2 amplified gastric carcinoma where the same methods are used for detection, albeit with different interpretation criteria. ${ }^{12}$ HER2 is of clinical importance also in lung carcinomas, although not as significant as in carcinomas of the breast and stomach. HER2 amplification in NSCLC is more common in adenocarcinomas of male smokers.4 Majority of the NGSAmp cases in this study were adenocarcinomas of male patients $(69 \%)$; however, we did not observe any difference between the NGSAmp and NGSNonAmp groups with regard to sex, age, or diagnosis.

HER2 amplification status in NSCLC is important for two different reasons: first, as one of the most common mechanisms of acquired resistance to TKIs and second, as a potential therapy target. ${ }^{13,14}$ Currently, several clinical trials are investigating the role of HER2-directed antibodies (trastuzumab, pertuzumab) as well as HER2-targeting TKIs (afatinib, dacomitinib, and neratinib). ${ }^{15}$ There are conflicting results on the therapy success of anti-HER2 monoclonal antibodies in NSCLC. ${ }^{6,16-18}$ While clinical studies on amplified HER2 as a therapy target are proceeding, it is not yet certain what method is suitable for evaluating HER2 amplification in NSCLC. With the increasing utilization of NGS panels in pathology laboratories, HER2 amplifications in NSCLC, along with other gene amplifications, have started to be observed by pathologists. However, there is not enough information on the correlation of CNV as determined by NGS and other techniques that have been traditionally used for HER2 detection in pathology laboratories. Therefore, unlike breast and gastric cancer, the HER2 amplification criteria have not been defined for lung carcinoma. There are some studies in the literature on HER2 amplification in NSCLC, performed with different ISH techniques, and HER2 amplification rates vary between $1.8 \%$ and $53 \%$. There are also some studies using immunohistochemistry to evaluate HER2 expression in NSCLC, according to which the incidence of $3+$ HER2 positivity, that has a

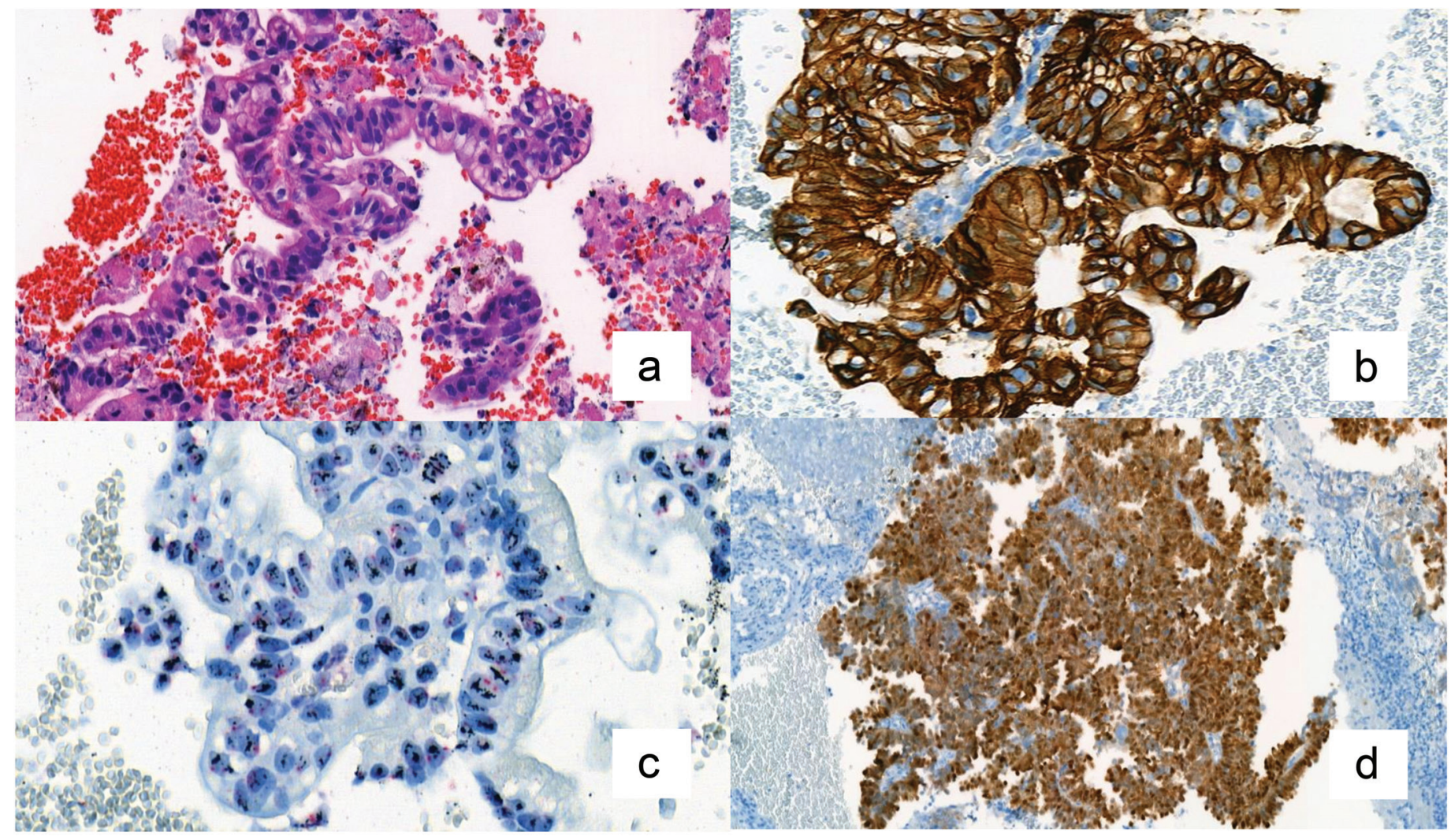

FIG. 1. a-d. Example of a NGS-HER2 amplified (a-c) and non-amplified (d) tumour: a-c: (Case number 2) Fold change of HER2 gene was found to be 8.81 by NGS for this case (a) HE (b) Complete membranous staining was observed in nearly all tumor cells (3+) HER2. (c) Dual-color SISH showed grouped HER2 signals, HER-2/neu probe is labeled in black and chromosome 17 enumeration probe is labeled in red. Slides were scanned with a digital scanner (Panoramic 250 Flash III, 3DHISTECH Ltd., Hungary) in 40× objective and photographed with CaseViewer 1.4 (3DHISTECH Ltd., Hungary) in 40× magnification. (D) (Case number 7) Membranous staining was not observed in this case; staining was mainly nuclear and cytoplasmic. HER2/CEP 17 was 2.17 in this case. Slides were scanned with a digital scanner (Panoramic 250 Flash III, 3DHISTECH Ltd., Hungary) in $40 \times$ objective and photographed with CaseViewer 1.4 (3DHISTECH Ltd., Hungary) in 5×(d) magnification. NGS, next-generation sequencing; SISH, silver in situ hybridization; HER2, Human epidermal growth factor 2. 
high correlation with HER2 amplification in the breast carcinoma context, varies from $0.6 \%$ to $33 \%$ of NSCLC cases. 16,18 A metaanalysis showed that HER2 overexpression determined by IHC is significantly associated with a poor prognosis in lung cancer. ${ }^{19}$ However, there is no previous study in the literature correlating NGS results with ISH and IHC on NSCLC patients, to the best of our knowledge.

HER2 amplification rate was found to be $12.8 \%$ by NGS in the current series, being quite higher than another NGS study stating the amplification rate as $1.3 \% .{ }^{20}$ When the lung carcinoma data from cBioPortal which covers 3864 NSCLC and adenocarcinomas is reviewed, it can be seen that HER2 amplification rate is about $1.9 \%$ in this database. ${ }^{21}$ Differences in the technique and calculation methods may have led to those discordant results. In our series, inter-method agreement was very good between HER2 FC by NGS and HER2/CEP17 ratio by SISH. However, there are $3 \mathrm{SISH}$ positive cases $(23 \%)$ within the NGSNonAmp group. NGS has the advantage of detecting high-level copy number gains in multiple genes simultaneously in addition to sequence variant detection. ${ }^{22}$ NGS is a very successful method in detecting many types of mutations such as point mutations, small indels, and small duplications; however, its sensitivity and specificity in $\mathrm{CNV}$ detection are controversial. ${ }^{23}$ The most important limitations of NGS are the difficulty of detecting low-level copy number gains and high-level amplifications in specimens with low neoplastic cell percentages, where in situ methods are recommended to either exclude or confirm the presence of copy number gains. ${ }^{22}$

Immunohistochemically definitive or equivocal HER2 positivity (stated as $3+$ or $2+$, or just positive) rate varies between 0.6 and $70 \%$ in the previous studies. There were 8 positive or equivocal $(3+/ 2+)$ cases among our 23 cases that were examined by IHC; rate being $50 \%$ and $23 \%$ in NGSAmp and NGSNonAmp groups, respectively. As nearly half (10/23) of immunohistochemically investigated cases were in the NGSAmp group, the overall $34.7 \%$ immunohistochemical positivity found in this study was probably higher than the real incidence. There are conflicting results in the literature on the correlation between immunohistochemical HER2 expression and amplification by ISH in NSCLC. There were some studies finding correlation ${ }^{4,7,24-26}$, and some studies stated that correlation was limited to $2+/ 3+$ cases. $^{18,27,28}$ On the other hand, poor correlation had been previously noted in some other studies, similar to our findings. ${ }^{29}$ Furthermore, according to our results, the difference between NGSAmp and NGSNonAmp groups with regard to immunohistochemical HER2 expression was not significant. Discordance between amplification/CNV detection and protein overexpression can be expected, as gene amplification is just one of the many mechanisms by which a gene is overexpressed. Expression of a gene can also be increased by mechanisms such as histone modifications, promoter demethylation, methylation of the gene, and changes in enhancer and silencer regions.

In this study, we observed nuclear staining in 5 of the 23 immunohistochemically stained NSCLCs. Nuclear/cytoplasmic staining which is quite unusual in breast carcinomas is also described by other authors; however, its incidence or meaning is not known. ${ }^{25}$ It is well known that immunohistochemical staining with the anti HER2/neu clone 4B5, which is used in the present study, can produce cytoplasmic and nuclear staining of normal gastric mucosa and more infrequently of neoplastic cells in gastric carcinoma. ${ }^{30}$ Unusual HER2 staining patterns are encountered in other organ carcinomas. There are several reports showing evidence of poor prognosis of HER2 cytoplasmic overexpression in colorectal and endometrial carcinomas. ${ }^{31,32}$ Although the mechanism underlying cytoplasmic and nuclear staining is currently unknown, two mechanisms among others can be speculated. The first one being nonspecific staining due to cross reaction and the second mechanism could be a mutation concerning the signal peptide of the HER2 gene, located at the 1-22 position of the protein, determining the path of the HER2 protein within the cell. ${ }^{21}$ Detection of mutations corresponding to this region of the protein (signal sequence) and detection of HER2 mRNA levels would potentially clarify this situation. However, we cannot comment on this, as our NGS panel did not cover the first exon of HER2 gene, where signal sequence is located. HER2 amplification is not an exclusive event in NSCLC. HER2 amplification was often accompanied by other mutations, and it was detected as the single molecular change in only $2 \%$ of our cohort. In a previous study, unique focal HER2 amplifications were identified in the oncogene-negative pulmonary carcinomas, using GISTIC (Genomic Identification of Significant Targets in Cancer) analysis. ${ }^{2}$ In that study, HER2 amplifications in otherwise oncogene-negative lung adenocarcinomas, which were found in $0.9 \%$ of their cohort, were suggested as driver events. ${ }^{2}$ In our study, EGFR and FGFR 1 amplifications were detected in $116(45 \%)$ and $35(13.6 \%)$ of the 256 cases, respectively. HER 2 and EGFR were co-amplified in 25 cases $(P<.001)$. FGFR1 and HER2 were co-amplified in 16 cases $(P<.001)$ by NGS. There were a few studies in the literature performed by Fluorescence in situ hybridization (FISH), showing that the copy number change in EGFR and HER2 genes shows correlation. ${ }^{33,34}$ However, there is no such previous observation regarding FGFR1 and HER2 correlation. As HER2, EGFR, and FGFR1 genes are located on separate chromosomes, 17q12, 7p11.2, and 8p.11.23, respectively, the mechanism of co-amplification remains to be solved.

The limitation of our study was the small number of cases that could be examined with IHC and SISH. Also, HER2 protein overexpression could not be confirmed by mRNA levels and other proteomic methods such as Western blotting.

NGS is now considered the preferable technique in searching multiple therapy targets in human tumors, and it is possible to evaluate the CNV of genes, including HER2, by NGS. However, there is not adequate knowledge on how to transfer this information to the management of therapy. Although there is a very good inter-method agreement between HER2 CNV and HER2 amplification by ISH, there is a considerable number of discordant cases, and not all ISH amplified cases are amplified by NGS. We hope that these ambiguous aspects may be solved 
by studies with a larger sample size. The small sample size of the present study due to the rarity of HER2 amplification in lung carcinomas and inadequacy of small tumor specimens is the major limitation of the present study. In the post hoc power analysis, power rate was calculated as 0.70 . Higher values can be reached in future studies with a larger sample size.

As an interesting finding, some NGS or ISH amplified cases do not show HER2 expression by IHC, which suggests that amplified gene is not reflected to protein synthesis. The mechanisms behind this condition may be related to the numerous control steps in the regulation of gene expression. Previous studies and our data show that HER2 gene abnormality in pulmonary carcinoma is not comparable to that in breast carcinoma, and the algorithms used in breast carcinoma do not apply to the lung. Larger-scale studies evaluating mRNA and protein levels are needed to understand the meaning of HER2 amplification detected by NGS in pulmonary carcinoma. Until the effects of HER2 amplification on HER2 protein are well understood and specific algorithms are defined, NSCLC cases that are found to be amplified by NGS should be verified by additional methods. It may be speculated that protein-based methods like IHC may have potential in decision-making for HER2 targeted therapy, as the target of the available drugs is the HER2 protein.

Ethics Committee Approval: Ethics committee approval was received from the Ethics Committee of Ankara University School of Medicine (approval number: I3-160-20).

Patient Consent for Publication: Written informed consent was obtained from the patients.

Data Sharing Statement: The data that support the findings of this study are available from the corresponding author upon reasonable request.

Author Contributions: Data Collection and/or Processing - H.K., H.Ö., S.D.S.; Analysis and/or Interpretation - H.Ö., D.K.; Literature Review - H.K., S.D.S; Writing - H.K., H.Ö., D.K., S.D.S.

Acknowledgments: The authors thank Yasemin Şahin, $\mathrm{PhD}$ for technical assistance, Tolga Bağlan, MD for case selection and İrem Kar, for statistical analyses.

Conflict of Interest: The authors have no conflict of interest to declare.

Funding: The authors declared that this study has received no financial support.

Supplementary: https://balkanmedicaljournal.org/uploads/pdf/supplenatry-table-1.pdf

\section{REFERENCES}

1. WilliamD, Travis EB, Burke AP, Marx A, Andrew GN. In: Bosman ESJFT, Lakhani SR, Ohgaki H, eds. WHO Classification of Tumours of the Lung, Pleura, Tyhmus and Heart. Tumours of the lung. 4th ed. Lyon, France: International Agency for Research on Cancer (IARC); 2015.

2. Cancer Genome Atlas Research Network. Comprehensive molecular profiling of lung adenocarcinoma. Nature. 2014;511:543-550. [CrossRef]

3. Rakha EA, Ellis IO, Horii R, et al. Invasive breast carcinoma: general overview. In: WHO Classification of Tumours Editorial Board, eds. Breast Tumours, WHO Classification of Tumours. 5th ed. Lyon, France: International Agency for Research on Cancer (IARC); 2019:82-101.

4. Li BT, Ross DS, Aisner DL, et al. HER2 amplification and HER2 mutation are distinct molecular targets in lung cancers. J Thorac Oncol. 2016;11:414-419. [CrossRef]

5. Heinmöller P, Gross C, Beyser K, et al. HER2 status in non-small cell lung cancer: results from patient screening for enrollment to a phase II study of Herceptin. Clin Cancer Res. 2003;9:5238-5243. [CrossRef]
6. Hotta K, Aoe K, Kozuki T, et al. A phase II study of trastuzumab emtansine in HER2-positive non-small cell lung cancer. J Thorac Oncol. 2018;13:273-279. [CrossRef]

7. Suzuki M, Shiraishi K, Yoshida A, et al. HER2 gene mutations in non-small cell lung carcinomas: concurrence with Her2 gene amplification and Her2 protein expression and phosphorylation. Lung Cancer. 2015;87:14-22. [CrossRef]

8. Mazières J, Peters S, Lepage B, et al. Lung cancer that harbors an HER2 mutation: epidemiologic characteristics and therapeutic perspectives. $J$ Clin Oncol. 2013;31:1997-2003. [CrossRef]

9. Visscher DW, Yadrandji S, Tabaczka P, Kraut M, Sarkar FH. Clinicopathologic analysis of k-ras, p53, and ERBB-2 gene alterations in pulmonary adenocarcinoma. Diagn Mol Pathol. 1997;6:64-69. [CrossRef]

10. Wolff AC, Hammond MEH, Allison $\mathrm{KH}$, et al. Human epidermal growth factor receptor 2 testing in breast cancer: American Society of Clinical Oncology/College of American Pathologists clinical practice guideline focused update. J Clin Oncol. 2018;36:2105-2122. [CrossRef]

11. Lokuhetty D, White V, Watanabe R, Cree I. WHO Classification of Breast Tumours. Geneva: WHO; 2019.

12. Abrahao-Machado LF, Scapulatempo-Neto C. HER2 testing in gastric cancer: an update. World J Gastroenterol. 2016;22:4619-4625. [CrossRef]

13. Takezawa K, Pirazzoli V, Arcila ME, et al. HER2 amplification: a potential mechanism of acquired resistance to EGFR inhibition in EGFR-mutant lung cancers that lack the second-site EGFRT790M mutation. Cancer Discov. 2012;2:922-933. [CrossRef]

14. Yu HA, Arcila ME, Rekhtman N, et al. Analysis of tumor specimens at the time of acquired resistance to EGFR-TKI therapy in 155 patients with EGFR-mutant lung cancers. Clin Cancer Res. 2013;19:2240-2247. [CrossRef]

15. Mar N, Vredenburgh JJ, Wasser JS. Targeting HER2 in the treatment of non-small cell lung cancer. Lung Cancer. 2015;87:220-225. [CrossRef]

16. Langer CJ, Stephenson P, Thor A, Vangel M, Johnson DH, Eastern Cooperative Oncology Group Study 2598. Trastuzumab in the treatment of advanced non-smallcell lung cancer: is there a role? Focus on Eastern Cooperative Oncology Group study 2598. J Clin Oncol. 2004;22:1180-1187. [CrossRef]

17. Clamon G, Herndon J, Kern J, et al. Lack of trastuzumab activity in nonsmall cell lung carcinoma with overexpression of erb-B2: 39810: a phase II trial of cancer and leukemia group B. Cancer. 2005;103:1670-1675. [CrossRef]

18. Gatzemeier U, Groth G, Butts C, et al. Randomized phase II trial of gemcitabinecisplatin with or without trastuzumab in HER2-positive non-small-cell lung cancer. Ann Oncol. 2004;15:19-27. [CrossRef]

19. Liu L, Shao X, Gao W, et al. The role of human epidermal growth factor receptor 2 as a prognostic factor in lung cancer: a meta-analysis of published data. $J$ Thorac Oncol. 2010;5:1922-1932. [CrossRef]

20. Lee K, Jung HA, Sun JM, et al. Clinical characteristics and outcomes of non-small cell lung cancer patients with HER2 alterations in Korea. Cancer Res Treat. 2020;52:292300. [CrossRef]

21. cBioPortal. cBioPortal for cancer genomics [internet]. Available at: https://www. cbioportal.org/.

22. Eijkelenboom A, Tops BBJ, van den Berg A, et al. Recommendations for the clinical interpretation and reporting of copy number gains using gene panel NGS analysis in routine diagnostics. Virchows Arch. 2019;474:673-680. [CrossRef]

23. Yao R, Yu T, Qing Y, Wang J, Shen Y. Evaluation of copy number variant detection from panel-based next-generation sequencing data. Mol Genet Genom Med. 2019;7:e00513. [CrossRef]

24. Yoshizawa A, Sumiyoshi S, Sonobe M, et al. HER2 status in lung adenocarcinoma: a comparison of immunohistochemistry, fluorescence in situ hybridization (FISH), dual-ISH, and gene mutations. Lung Cancer. 2014;85:373-378. [CrossRef]

25. Kim EK, Kim KA, Lee CY, Shim HS. The frequency and clinical impact of HER2 alterations in lung adenocarcinoma. PLoS One. 2017;12:e171280. [CrossRef]

26. Tan D, Deeb G, Wang J, et al. HER-2/neu protein expression and gene alteration in stage I-IIIA non-small-cell lung cancer: a study of 140 cases using a combination of high throughput tissue microarray, immunohistochemistry, and fluorescent in situ hybridization. Diagn Mol Pathol. 2003;12:201-211. [CrossRef]

27. Cox G, Vyberg M, Melgaard B, Askaa J, Oster A, O’Byrne KJ. Herceptest: HER2 expression and gene amplification in non-small cell lung cancer. Int $J$ Cancer. 2001;92:480-483. [CrossRef] 
28. Kuyama S, Hotta K, Tabata M, et al. Impact of HER2 gene and protein status on the treatment outcome of cisplatin-based chemoradiotherapy for locally advanced nonsmall cell lung cancer. J Thorac Oncol. 2008;3:477-482. [CrossRef]

29. Nakamura H, Saji H, Ogata A, et al. Correlation between encoded protein overexpression and copy number of the HER2 gene with survival in non-small cell lung cancer. Int J Cancer. 2003;103:61-66. [CrossRef]

30. Rüschoff J, Dietel M, Baretton G, et al. HER2 diagnostics in gastric cancer-guideline validation and development of standardized immunohistochemical testing. Virchows Arch. 2010;457:299-307. [CrossRef]

31. Osako $\mathrm{T}$, Miyahara $\mathrm{M}$, Uchino $\mathrm{S}$, Inomata $\mathrm{M}$, Kitano $\mathrm{S}$, Kobayashi $\mathrm{M}$. Immunohistochemical study of c-erbB-2 protein in colorectal cancer and the correlation with patient survival. Oncology. 1998;55:548-555. [CrossRef]

32. Demirbaş S, Sücüllü I, Yildirim S, Celenk T. Influence of the c-erb B-2, nm23, bcl-2 and p53 protein markers on colorectal cancer. Turk J Gastroenterol. 2006;17:13-19.
33. Varella-Garcia M, Mitsudomi T, Yatabe Y, et al. EGFR and HER2 genomic gain in recurrent non-small cell lung cancer after surgery: impact on outcome to treatment with gefitinib and association with EGFR and KRAS mutations in a Japanese cohort. J Thorac Oncol. 2009;4:318-325. [CrossRef]

34. Cappuzzo F, Varella-Garcia M, Shigematsu H, et al. Increased HER2 gene copy number is associated with response to gefitinib therapy in epidermal growth factor receptor-positive non-small-cell lung cancer patients. J Clin Oncol. 2005;23:50075018. [CrossRef]

35. Hirashima N, Takahashi W, Yoshii S, Yamane T, Ooi A. Protein overexpression and gene amplification of c-erb B-2 in pulmonary carcinomas: a comparative immunohistochemical and fluorescence in situ hybridization study. Mod Pathol. 2001;14:556-562. [CrossRef]

36. Hirsch FR, Varella-Garcia M, Franklin WA, et al. Evaluation of HER-2/neu gene amplification and protein expression in non-small cell lung carcinomas. Br J Cancer. 2002;86:1449-1456. [CrossRef] 